\title{
Dynamic of biocontrol agents against Botrytis cinerea on grape leaves
}

\section{Dinámica de agentes de biocontrol contra Botrytis cinerea en hojas de vid}

Yara Suhan Juárez-Campusano, María del Socorro Chávaro-Ortíz, Rocío Crystabel López-González, Juan Ramiro Pacheco-Aguilar*

Facultad de Química, Universidad Autónoma de Querétaro. Cerro de las Campanas, s/n, Las Campanas, 76010 Santiago de Querétaro, Qro, Mexico.

${ }^{*}$ Corresponding author

E-mail address: juanramiro29@yahoo.com.mx (J. R. Pacheco-Aguilar)

Article history:

Received: 15 December 2019 / Received in revised form: 7 April 2020 / Accepted: 21 April 2020 / Published online: 4 May 2020.

https://doi.org/10.29267/mxib.2020.5.2.70

\begin{abstract}
The survival of biocontrol agents in plant tissues is an important characteristic for bioprotection against phytopathogenic organisms. In the present work, the survival of two biocontrol agents on grape leaves, Bacillus methylotrophicus FR4B12 and Metschnikowia pulcherrima NB9, was evaluated, as well as their interaction against Botrytis cinerea, a phytopathogen causing gray mold disease on grapes. To that end, grape leaves of the Cabernet Sauvignon and Syrah varieties were inoculated with $10^{4}$ spores of $B$. cinerea, followed by $10^{7}$ cells of each antagonist. Additionally, as a control group, individual leaves were inoculated with each of the microorganisms tested. The leaves were kept in a moist chamber, and were collected at 0,48 and $96 \mathrm{~h}$ to determine the microbial populations. The results showed $B$. methylotrophicus to be the biocontrol agent with the best performance in terms of colonization and survival on grape leaves. B. methylotrophicus increased its population densities in a range of $2.4 \times 10^{3}-6.9 \times 10^{3} \mathrm{UFC} \mathrm{cm}^{-2}$ on both varieties of leaves at $96 \mathrm{~h}$, which was observed on both sole inoculation and co-inoculation with $B$. cinerea. As a consequence, the pathogenic activity on the leaves was reduced.
\end{abstract}


Keywords: Bacillus methylotrophicus, epiphytic populations, gray mold, Metschnikowia pulcherrima, microbial antagonism.

\section{RESUMEN}

La supervivencia de los agentes de biocontrol en los tejidos vegetales es una característica importante en la bioprotección contra organismos fitopatógenos. En el presente trabajo se evaluó la supervivencia de dos agentes de biocontrol, Bacillus methylotrophicus FR4B12 y Metschnikowia pulcherrima NB9, sobre hojas de vid, así como su interacción contra Botrytis cinerea, fitopatógeno causante del mildiú gris en uva. Para ello, se inocularon hojas de vid de las variedades cabernet sauvignon y syrah con $10^{4}$ esporas de $B$. cinerea, seguidas por $10^{7}$ células de cada uno de los antagonistas. Como controles, se inocularon hojas individualmente con cada uno de los microorganismos ensayados. Las hojas se mantuvieron en cámara húmeda, siendo colectadas a las 0,48 y $96 \mathrm{~h}$ para determinar la densidad de las poblaciones microbianas. Los resultados muestran que $B$. methylotrophicus fue el agente de biocontrol con el mejor desempeño en cuanto a colonización y supervivencia en hojas de vid, registrando a las $96 \mathrm{~h}$ incrementos en la densidad poblacional dentro del rango de $2.4 \times 10^{3}$ a $6.9 \times 10^{3}$ UFC $\mathrm{cm}^{-2}$ para ambas variedades de vid, tanto en la inoculación individual como durante la interacción con $B$. cinerea. En consecuencia, se registró una reducción en la actividad patogénica sobre las hojas.

Palabras clave: Bacillus methylotrophicus, poblaciones epífitas, mildiú gris, Metschnikowia pulcherrima, antagonismo microbiano.

\section{INTRODUCCIÓN}

Botrytis cinerea es un hongo fitopatógeno que causa la podredumbre gris en un amplio rango de hospederos de frutas y hortalizas, tales como fresa, manzana, col, zanahoria, pepino, berenjena, lechuga, frijol, mostaza, arroz, chícharo, pimiento y jitomate, entre otros (Pande et al., 2006; Keller, 2015). En el estado de Querétaro, el control de esta enfermedad es de particular interés, pues afecta el cultivo de la vid, el cual ha cobrado gran importancia debido al aumento de ingresos económicos conseguidos a través de la industria vinícola y el enoturismo (AVQ, 2011; Amo, 2018).

Para el control del este patógeno, se han desarrollado agroquímicos del tipo de anilinopirimidinas, fenilpirroles, hidroxianilinas y benzimidazoles entre otros, cuyo modo de acción está dirigido a inhibir la germinación de esporas, el crecimiento micelial y la reproducción del hongo (Rosslenbroich \& Stuebler, 2000). El control químico ha representado a nivel global una inversión de más de 15 millones de dólares anuales (Khazaeli et al., 2012) sin obtener resultados del todo favorables debido a la múltiple resistencia que ha generado Botrytis, además de que el abuso 
de estos pesticidas ha provocado un impacto negativo sobre los ecosistemas agrícolas y la salud humana (Rupp et al., 2007; Guerrero et al., 2019). Por lo tanto, es necesario el empleo de métodos de control ambientalmente más sostenibles que disminuyan o sustituyan el uso de agroquímicos, reduciendo con ello la huella ecológica (Zhang et al., 2017).

Entre los métodos alternativos, el control biológico ha sido una de las opciones emergentes que ha demostrado ser eficaz. Dicho control se basa en el uso de microorganismos benéficos antagonistas, los cuales producen moléculas que inhiben el crecimiento y actividad de los microorganismos fitopatógenos mediante la producción de moléculas antimicrobianas y la competencia por espacio y nutrientes (Dukare et al., 2018). Diversos estudios sobre ecología microbiana han documentado la presencia de bacterias, levaduras y hongos en los órganos de las plantas, los cuales juegan un papel importante en la defensa contra patógenos, por lo que son candidatos efectivos para el control de enfermedades (Bolívar et al., 2019). Dentro de las bacterias más estudiadas y comercializadas se encuentra Bacillus, debido a que produce un amplio rango de moléculas que limita el desarrollo de diversos fitopatógenos. Además, produce esporas, lo que le permite sobrevivir bajo condiciones ambientales adversas (Silva et al., 2019). Otro grupo de microorganismos estudiados, pero menos comercializados como agentes de control biológico, son las levaduras, cuyas aplicaciones se han enfocado principalmente al control de hongos patógenos en frutos poscosecha. Entre los géneros más estudiados se encuentran los siguientes: Metschnikowia, Pichia, Cryptococcus y Aureobasidium (Ferraz et al., 2019).

La capacidad de colonizar tejidos vegetales e integrarse a las comunidades microbianas presentes in situ es una característica de particular interés que se busca en los agentes de biocontrol, ya que de ello depende en gran medida el éxito de la bioprotección (Wei et al., 2016).

El objetivo del presente trabajo fue evaluar la supervivencia en hojas de vid (órgano de reservorio de $B$. cinerea) de Bacillus methylotrophicus FR4B12 y Metschnikowia pulcherrima NB9 (cepas antagonistas con potencial de biocontrol), así como también determinar los cambios en las poblaciones cuando son coinoculados con dos cepas de $B$. cinerea en un ensayo de biocontrol de infección.

\section{MATERIALES Y MÉTODOS}

\subsection{Material biológico}

Los agentes de biocontrol empleados en el presente estudio fueron los siguientes: Bacillus methylotrophicus FR4B12, una cepa aislada de frutos de árboles de manzano de la variedad "Royal Gala" de un huerto experimental establecido en el municipio de Amealco, Querétaro; y la levadura antagonista Metschnikowia pulcherrima NB9, que es una cepa aislada de la fermentación espontanea de un mosto de uva elaborado con frutos provenientes del viñedo "El Barreno" de San 
Juan del Río, Querétaro. Ambas cepas han mostrado, en ensayos in vitro (Fig. 1) e in vivo, inhibir el desarrollo de B. cinerea (López-González et al., 2019).

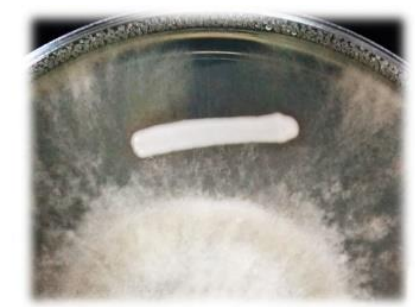

Cepa NB9

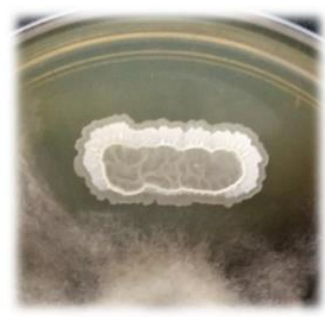

Cepa FR4B12

Fig. 1. Ensayo dual in vitro de confrontación de las cepas antagonistas de estudio contra Botrytis cinerea. NB9: M. pulcherrima, FR4B12: B. methylotrophicus.

Fig. 1. In vitro dual test of the study's antagonist strains against Botrytis cinerea. NB9: M. pulcherrima, FR4B12: B. methylotrophicus.

En cuanto a los patógenos, se emplearon las cepas de $B$. cinerea 133 y $B$. cinerea 155 , las cuales fueron aisladas de frutos de uva de las variedades syrah y merlot, respectivamente, provenientes de las vinícolas queretanas "Bodega De Cote" y "El Rosario". Su identificación fue realizada mediante la amplificación y secuenciación del marcado molecular ITS.

Para los ensayos de infección se emplearon hojas jóvenes de plantas de un año de edad de las variedades "cabernet sauvignon" y "syrah". Dichas plantas fueron obtenidas mediante propagación por estaca, y provienen de la vinícola "Cuna de Tierra" ubicada en Dolores Hidalgo, Gto.

\subsection{Preparación de los inóculos}

Para preparar los inóculos de biocontrol, se cultivaron la levadura NB9 y la bacteria FR4B12 en los medios NYDB ( $\mathrm{g} \mathrm{L}^{-1}$ : caldo nutritivo 8.0, extracto de levadura 2.0, glucosa 10.0) y caldo nutritivo $\left(8 \mathrm{~g} \mathrm{~L}^{-1}\right)$, respectivamente. Los cultivos fueron mantenidos en agitación constante durante $18 \mathrm{~h}$ a $30^{\circ} \mathrm{C} y$, al término, fueron centrifugados a $4000 \mathrm{rpm}$ por 20 min para obtener las pastillas celulares, las cuales fueron después resuspendidas en solución salina al $0.9 \%$ para realizar la cuenta celular en un hematocímetro con la ayuda de microscopio (Martínez et. al., 2013). Finalmente, la densidad fue ajustada a $1 \times 10^{7}$ células $\mathrm{mL}^{-1}$.

Para la preparación del inóculo de Botryitis, se cultivaron las cepas 133 y 155 en placas de medio NYDA (NYDB + $20 \mathrm{~g} \mathrm{~L}^{-1}$ de agar bacteriológico) hasta la esporulación (10 días). Posteriormente, las esporas fueron removidas de la superficie empleando una varilla estéril y una solución de Tween 20 al $0.5 \%$. El extracto fue filtrado con una gasa estéril para eliminar el micelio y recuperar las 
esporas (Petrasch et. al., 2019). Finalmente fueron ajustadas a una concentración de $1 \times 10^{4}$ esporas $\mathrm{mL}^{-1}$.

\subsection{Ensayos de inoculación de hojas de vid}

Para determinar el establecimiento de las poblaciones antagonistas y del patógeno sobre hojas de vid, estas fueron lavadas con una solución de Tween 20 al $0.5 \%$, dejándose secar en una campana de flujo laminar. Posteriormente, se inocularon por aspersión con $1 \mathrm{~mL}$ de cada uno de los antagonistas $1 \times 10^{7}$ células $\mathrm{mL}^{-1}$ (Arriaga et al., 2012; Pauluk-Correa et al., 2018) o con $1 \mathrm{~mL}$ de la mezcla 50 y 50 $\%$ de ambas cepas de Botrytis ( $1 \times 10^{4}$ esporas $\left.\mathrm{mL}^{-1}\right)$, según Whiteman \& Stewart (1998).

Para los ensayos de interacción antagonista vs patógeno, las hojas fueron primeramente inoculadas con $1 \mathrm{~mL}$ de la mezcla de ambas cepas de Botrytis (1×104 esporas $\left.\mathrm{mL}^{-1}\right)$, y después de 1 hora se realizó la inoculación de $1 \mathrm{~mL}$ del antagonista $\left(1 \times 10^{7}\right.$ células $\left.\mathrm{mL}^{-1}\right)$.

Adicionalmente, se consideraron hojas control sin inocular. Todos los tratamientos fueron puestos en cámara húmeda por $96 \mathrm{~h}$. Por cada uno de los tratamientos (antagonista, patógeno, interacción antagonista-patógena y control) fueron empleadas 5 hojas de cada variedad.

\subsection{Cuantificación de poblaciones microbianas}

Para determinar la persistencia de las poblaciones patógenas y antagonistas en las hojas, se realizaron muestreos utilizando un sacabocado estéril, obteniendo tres círculos de $7 \mathrm{~mm}$ de diámetro de tejido vegetal a diferentes tiempos $(0,48 \mathrm{y}$ $96 \mathrm{~h}$ ). Para recuperar las células, las muestras fueron inmersas en $1 \mathrm{~mL}$ de buffer de fosfatos y sonicadas por 20 min. Después, $100 \mu \mathrm{L}$ de esta solución de extracción fueron sembrados por triplicado en los siguientes medios: agar nutritivo + fluconazol (70 $\left.\mathrm{mg} \mathrm{L}^{-1}\right)$, YPDA ( $\mathrm{g} \mathrm{L}^{-1}$ : dextrosa 20.0, peptona 20.0, extracto de levadura 10.0, agar bacteriológico 20.0) + estreptomicina $\left(50 \mu \mathrm{g} \mathrm{L}^{-1}\right)$ y NYDA + rosa de bengala ( $50 \mathrm{mg} \mathrm{L}^{-1}$ ) para realizar los conteos de las poblaciones de bacterias, levaduras y hongos, respectivamente. Las cajas fueron incubadas a temperatura ambiente, realizando el conteo de las colonias después de 24 y $72 \mathrm{~h}$. Para el cálculo de la densidad de poblaciones, los resultados obtenidos de la cuenta viable fueron divididos entre el área del tejido muestreado para ser expresados como Unidades Formadoras de Colonias (UFC) por $\mathrm{cm}^{2}$ de hoja. 


\subsection{Análisis estadístico}

Los resultados obtenidos de las densidades de las poblaciones microbianas de cada tratamiento fueron transformados a escala logarítmica $\left(\log _{10} \mathrm{UFC} \mathrm{cm}^{-2}+1\right)$ y procesados en el programa estadístico INFOSTAT para ser sujetos a análisis de varianza (ANOVA) y prueba de comparación de medias Tukey $(p \leq 0.05)$ con la finalidad de buscar diferencias entre las poblaciones microbianas.

\section{RESULTADOS}

La Figura 2 muestra los cambios en la densidad poblacional de la bacteria antagonista $B$. methylotrophicus FR4B12 y del hongo fitopatógeno de $B$. cinerea en el ensayo de hojas de vid de la variedad cabernet sauvignon. Este comportamiento de las poblaciones fue similar en la variedad syrah, por lo que solo se presentará el gráfico de la primera variedad, pero se discutirán los resultados en las tablas para ambas variedades.

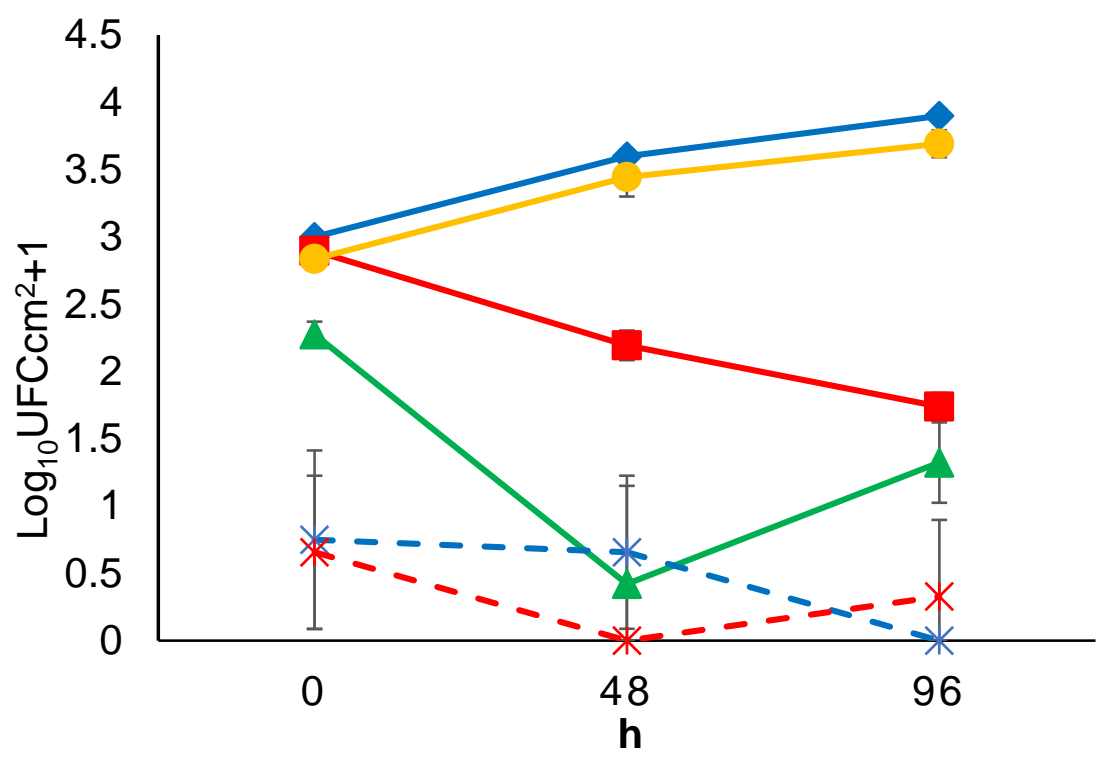

Fig. 2. Dinámica de las poblaciones microbianas en hojas de vid cabernet sauvignon. Densidad de bacterias después de la inoculación individual de $B$. methylotrophicus FR4B12 ( $\diamond$; en la interacción con B. cinerea (๑); densidad de hongos después de la inoculación individual de $B$. cinerea ( $\square$ ); en interacción con B. methylotrophicus FR4B12 ( $\mathbf{\Delta})$; bacterias (-ж-) y hongos (-x-) en hojas control.

Fig. 2. Dynamic of microbial populations in grape leaves Cabernet Sauvignon variety; bacterial densities after $B$. methylotrophicus FR4B12 inoculation ( $\diamond)$; in interaction with $B$. cinerea $(\bullet)$; fungal densities after $B$. cinerea inoculation $(\square)$; in interaction with $B$. methylotrophicus FR4B12 ( $\Delta$ ); bacterial (-ж-) and fungal (-X-) on control leaves. 
La inoculación individual de B. methylotrophicus FR4B12 en las hojas de vid de ambas variedades tuvo un incremento en la densidad poblacional en un rango de $5.4 \times 10^{3}$ a $6.9 \times 10^{3}$ UFC $\mathrm{cm}^{-2}$ en un lapso de $96 \mathrm{~h}$. Lo mismo ocurrió cuando se realizó la coinoculación con las cepas de $B$. cinerea; sin embargo, estos incrementos disminuyeron en un rango del 39 al $55 \%$, en comparación con las densidades encontradas cuando la cepa FR4B12 fue inoculada de manera individual, lo que podría indicar una interacción microbiana del antagonista con el hongo fitopatógeno (Tabla 1). Cabe mencionar que en las hojas control sin inocular también se detectaron poblaciones bacterianas; no obstante, estas representaron en promedio el $0.1 \%$ de la población final, lo que indica la capacidad de colonización de FR4B12.

Tabla 1. Análisis de la cuenta viable de la densidad de las poblaciones bacterianas a través de tiempo registradas en los tratamientos durante el ensayo en hojas de vid.

Table 1. Analysis of the viable count of population densities of bacterial communities over time in the grape leaves treatments.

\begin{tabular}{ccccc}
\hline Tiempo & $\begin{array}{c}\text { FR4B12 } \\
\text { CS }\end{array}$ & FR4B12 S & $\begin{array}{c}\text { FRAB12 vs } \\
\text { Botrytis CS }\end{array}$ & $\begin{array}{c}\text { FRAB12 vs } \\
\text { Botrytis S }\end{array}$ \\
\hline $\mathbf{0}$ & $3.00 \mathrm{a}^{*}$ & $3.17 \mathrm{a}$ & $2.84 \mathrm{a}$ & $2.93 \mathrm{a}$ \\
$\mathbf{4 8}$ & $3.60 \mathrm{~b}$ & $3.56 \mathrm{~b}$ & $3.44 \mathrm{~b}$ & $3.18 \mathrm{ab}$ \\
$\mathbf{9 6}$ & $3.90 \mathrm{c}$ & $3.84 \mathrm{c}$ & $3.69 \mathrm{~b}$ & $3.52 \mathrm{~b}$
\end{tabular}

Lo valores son el promedio de tres repeticiones expresado como el Log10 (UFCcm $\left.{ }^{-2}+1\right)$. *Medias con letras iguales en cada columna no son estadísticamente diferentes (Tukey, 0.05); FR4B12: inoculación con B. methylotrophicus; CS: cabernet sauvignon; S: syrah.

Con respecto a la inoculación individual de la levadura Metschnikowia pulcherrima NB9, podemos observar en la Figura 3 un rápido incremento en la densidad poblacional a partir de las $48 \mathrm{~h}$, cuyos valores se mantuvieron similares hasta las $96 \mathrm{~h}$. Estos incrementos registrados fueron del orden de $1.3 \times 10^{3}$ a $1.9 \times 10^{3}$ UFC $\mathrm{cm}^{-2}$ para ambas variedades de vid (Tabla 2). Dichos incrementos, comparados con los observados en $B$. methylotrophicus FR4B12, sólo representaron en promedio el $26 \%$ en ambas variedades de hojas.

Adicionalmente, podemos mencionar que las hojas control sin inocular mostraron una alta densidad de levaduras propias del tejido vegetal, las cuales no fueron removidas con la solución Tween 20. Estas poblaciones tuvieron una densidad promedio de $4.3 \times 10^{2}$ UFC $\mathrm{cm}^{-2}$ al término de las $96 \mathrm{~h}$, representando en proporción el $48 \%$ de la densidad total de las levaduras encontradas cuando se inoculó NB9. 
Tabla 2. Análisis de la cuenta viable de la densidad de las poblaciones de levaduras a través de tiempo registradas en los tratamientos durante el ensayo en hojas de vid.

Table 2. Analysis of the viable count of population densities of yeasts communities over time in the grape leaves treatments.

\begin{tabular}{ccccc}
\hline Tiempo & NB9 CS & NB9 S & $\begin{array}{c}\text { NB9 vs } \\
\text { Botrytis CS }\end{array}$ & $\begin{array}{c}\text { NB9 vs } \\
\text { Botrytis S }\end{array}$ \\
\hline $\mathbf{0}$ & $1.90 \mathrm{a}$ & $1.98 \mathrm{a}$ & $1.78 \mathrm{a}$ & $1.69 \mathrm{a}$ \\
$\mathbf{4 8}$ & $3.12 \mathrm{~b}$ & $3.01 \mathrm{~b}$ & $2.75 \mathrm{~b}$ & $2.58 \mathrm{~b}$ \\
$\mathbf{9 6}$ & $3.30 \mathrm{~b}$ & $3.16 \mathrm{~b}$ & $2.95 \mathrm{~b}$ & $3.01 \mathrm{c}$
\end{tabular}

Lo valores son el promedio de tres repeticiones expresado como el Log10 $\left(\mathrm{UFCcm}{ }^{-2}+1\right)$. *Medias con letras iguales en cada columna no son estadísticamente diferentes (Tukey, 0.05); NB9: inoculación de con M. pulcherrima; CS: cabernet sauvignon; S: syrah.

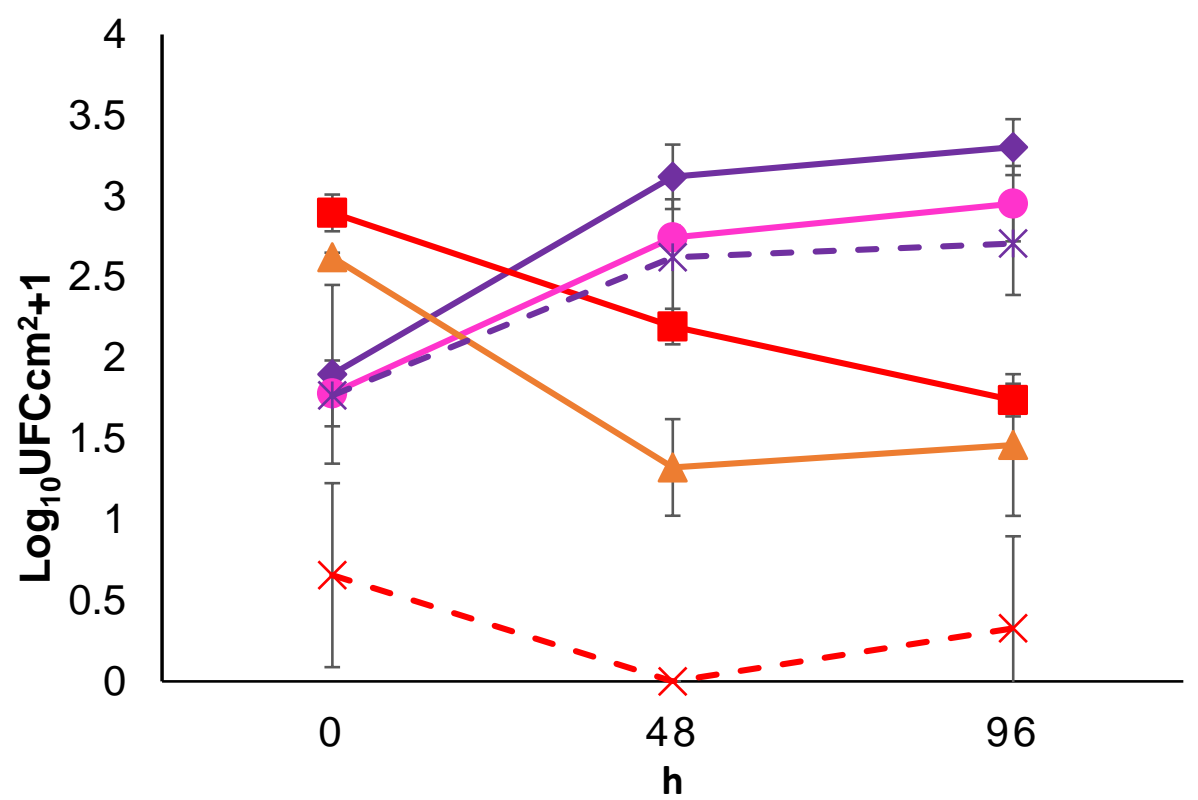

Fig. 3. Dinámica de las poblaciones microbianas en hojas de vid cabernet sauvignon. Densidad de levaduras después de la inoculación individual de $M$. pulcherrima NB9 (४); en interacción con $B$. cinerea (•); densidad de hongos después de la inoculación individual de $B$. cinerea ( $\square$ ); en interacción con $M$. pulcherrima NB9 ( $\triangle$ ); levaduras (-ж-) y hongos (-x-) en hojas control.

Fig. 3. Dynamic of microbial populations in grape leaves Cabernet Sauvignon variety. Yeast densities after M. pulcherrima NB9 inoculation (४); in interaction with $B$. cinerea ( $(\bullet)$; fungal densities after $B$. cinerea inoculation ( $\square)$; in interaction with M. pulcherrima NB9 ( $\triangle$ ); yeast (-ж-) and fungi (-x-) on control leaves. 
Finalmente, el análisis de las poblaciones de $B$. cinerea mostró que, cuando es inoculada individualmente en las hojas de vid, la densidad de sus poblaciones se

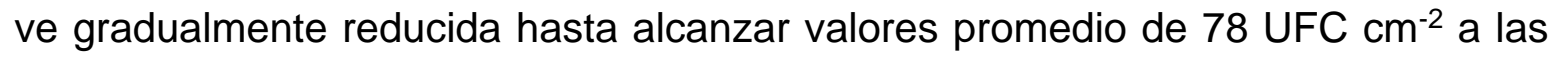
$96 \mathrm{~h}$. Un análisis estadístico comparativo de las densidades poblacionales de Botrytis a las $96 \mathrm{~h}$, donde se incluyeron todos los datos obtenidos de los tratamientos (con y sin inoculación de los antagonistas), mostró que no existen diferencias entre las densidades finales $(p \leq 0.05)$. Sin embargo, los análisis través del tiempo (Tabla 3) muestran que cuando Botrytis es confrontado con los antagonistas, sus densidades descienden en un $94 \%$ a las $48 \mathrm{~h}$, pero después se vuelven a restablecer las densidades poblacionales de hongos. Estos cambios no son observables cuando Botrytis se inocula individualmente en las hojas. Cabe mencionar que las hojas control sin inocular presentaron también densidades poblacionales de hongos, los cuales representaron en promedio el $7 \%$ de las poblaciones finales.

Tabla 3. Análisis de la cuenta viable de la densidad de las poblaciones de hongos a través de tiempo registradas en los tratamientos durante el ensayo en hojas de vid.

Table 3. Analysis of the viable count of population densities of fungal communities over time in the grape leaves treatments.

\begin{tabular}{|c|c|c|c|c|c|c|}
\hline Tiempo & $\begin{array}{l}\text { Botrytis } \\
\text { CS }\end{array}$ & $\begin{array}{c}\text { Botrytis } \\
\mathrm{S}\end{array}$ & $\begin{array}{l}\text { Botrytis vs } \\
\text { FR4B12 CS }\end{array}$ & $\begin{array}{l}\text { Botrytis vs } \\
\text { FR4B12 CS }\end{array}$ & $\begin{array}{c}\text { Botrytis vs } \\
\text { NB9 CS }\end{array}$ & $\begin{array}{c}\text { Botrytis vs } \\
\text { NB9 CS }\end{array}$ \\
\hline 0 & $2.90 \mathrm{c}$ & $2.41 \mathrm{~b}$ & $2.28 \mathrm{~b}$ & $2.00 \mathrm{~b}$ & $2.62 \mathrm{~b}$ & $2.37 \mathrm{~b}$ \\
\hline 48 & $2.19 b$ & $2.19 a b$ & $0.42 \mathrm{a}$ & $1.35 \mathrm{a}$ & $1.32 \mathrm{a}$ & $0.84 a$ \\
\hline 96 & $1.74 \mathrm{a}$ & $2.01 \mathrm{a}$ & $1.32 \mathrm{ab}$ & $1.57 \mathrm{ab}$ & $1.46 \mathrm{a}$ & $1.59 \mathrm{ab}$ \\
\hline
\end{tabular}

En la Fig. 4 se presentan los resultados de la infección con las cepas inoculadas de Botrytis, así como también el efecto de biocontrol cuando se coinocularon con las cepas FR4B12 y NB9, en donde se puede observar que la cepa de Bacillus fue la que redujo, en mayor medida, las lesiones causadas por Botrytis. Lo anterior debido, probablemente, a la interacción antagonista que provocó una reducción en la actividad patogénica de Botrytis. 


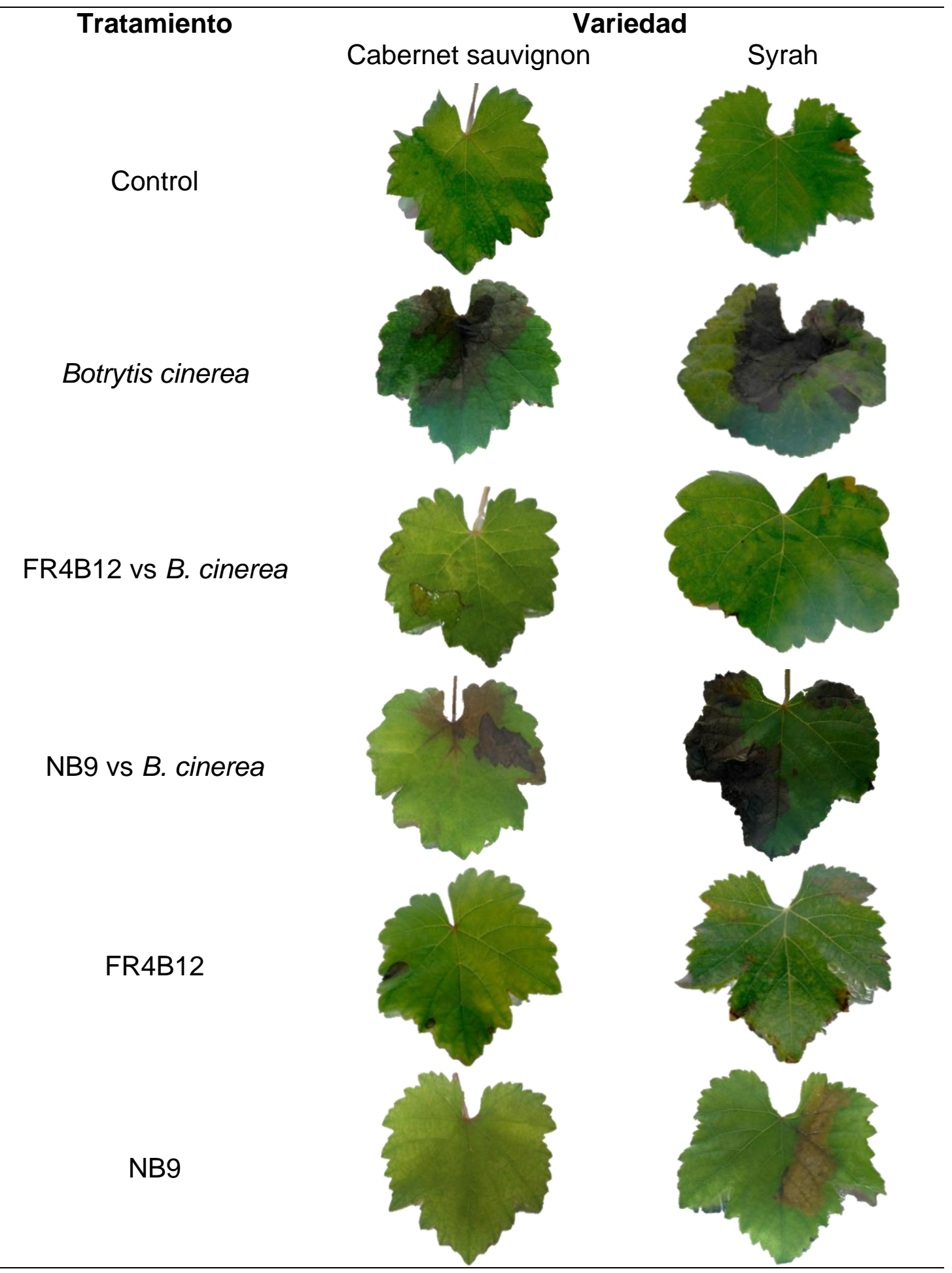

Fig. 4. Ensayos de inoculación en hojas de vid a las 96 h. FR4B12: inoculación con Bacillus methylotrophicus; NB9: inoculación con Metschnikowia pulcherrima. Fig. 4. Inoculation tests on grape leaves at 96 h. FR4B12: Bacillus methylotrophicus inoculation; NB9: Metschnikowia pulcherrima inoculation. 


\section{DISCUSIÓN}

La filósfera que comprende los tejidos aéreos de las plantas, a diferencia de la rizósfera, es una zona hostil para el desarrollo de los microorganismos. El viento, la radiación solar, los cambios repentinos de temperatura y humedad, así como la baja concentración de nutrimentos son solo algunos factores a los que deben enfrentarse los agentes de biocontrol para mantenerse viables (Perazzolli et al., 2014). En el presente trabajo, aunque fue realizado bajo condiciones de laboratorio, se determinó el establecimiento y el incremento de las poblaciones de los agentes de biocontrol en hojas de vid de las variedades cabernet sauvignon y syrah, siendo B. methylotrophicus FR4B12 el que mostró el mayor incremento $\left(10^{3}\right.$ UFC $\mathrm{cm}^{-2}$ ), alcanzando densidades de hasta $7.9 \times 10^{3} \mathrm{UFC} \mathrm{cm}^{-2}$ a las $96 \mathrm{~h}$, valores que se encuentran dentro del rango de la poblaciones bacterianas en vid. Cappelletti et al. (2016) y Vionnet et al. (2018) reportan densidades naturales bacterianas de $3 \times 10^{2}$ y $1.5 \times 10^{5}$ UFC $\mathrm{cm}^{-2}$ en los cultivares solaris y pinot noir, respectivamente, lo que parece indicar que, probablemente, las poblaciones de FR4B12 pudieran incrementarse si se extendiera el tiempo del ensayo. En cuanto al establecimiento de la levadura NB9, sus valores de densidad de $1.9 \times 10^{3}$ UFC $\mathrm{cm}^{-2}$ pudieran reflejar en sumativa las levaduras detectadas desde el principio en el tejido, aunque cabe mencionar que estas se encontraron en valores muy altos con respecto a lo reportado por Lúquez et al. (2007), quienes cuantificaron en hojas jóvenes y maduras de la variedad malbec valores promedios de $20 \mathrm{UFC} \mathrm{cm}$ 2. Con respecto a las poblaciones fúngicas, en el ensayo de Botrytis, la densidad final se detectó en 78 UFC cm$~_{-2}$ como valor promedio para todos los tratamientos con y sin los agentes de biocontrol, valor similar al reportado por Cappeppetti et al., (2016) quienes cuantificaron en la variedad pinot noir las poblaciones totales de hongos, registrando un valor promedio de $71 \mathrm{UFC} \mathrm{cm}^{-2}$. La permanencia y viabilidad de los agentes de biocontrol se ha vinculado con su capacidad para formar biofilm sobre los tejidos de las plantas, lo cual les da ventajas sobre otros grupos microbianos (Pandin et al., 2017). Esta capacidad ya ha sido demostrada para la mayoría de las especies del género Bacillus (Aleti et al., 2016), así como para la levadura M. pulcherrima (Gianotti et al., 2001). En cuanto a la interacción con $B$. cinerea, en ambos casos, las densidades microbianas obtenidas de bacterias y levaduras fueron menores a las encontradas cuando se inocularon de forma individual a los agentes de biocontrol. Estos cambios en la dinámica y estructura de las poblaciones microbianas, que fueron observados en las hojas de vid, debido a la aplicación ya sea de los agentes de biocontrol o del fitopatógeno, coinciden con lo encontrado por Zhang et. al. (2008) en ensayos en cultivo de chile (Capsicum annuum L.), en donde se observó un cambio en la presencia tanto para la población de bacterias como para las de hongos cuando se aplicó sobre las hojas la cepa de biocontrol Bacillus thuringiensis. También esto ha sido documentado en plantas de tabaco (Nicotiana tabacum), en donde, al aplicar la cepa antagonista Bacillus amyloliquefaciens en las raíces, se encontró que aumentaban las poblaciones de bacterias, las cuales incluía algunos géneros 
relacionados con promoción de crecimiento, como Pseudomonas, Mesorhizobium, Acinetobacter, Bradyrhizobium y Azospirillum (Wu et al., 2016). De forma contraria, en un modelo en papa (Solanum tuberosum) se observó que, al inocular una cepa antagonista del género Bacillus, se estimuló el crecimiento del fitopatógeno Fusarium coeruleum (Cray et al., 2016). Esto demuestra que la introducción de agentes de biocontrol puede ocasionar cambios en la comunidad microbiana establecida, lo cual puede traer consecuencias tanto positivas como negativas para la planta hospedera. Por otro lado, la cepa $B$. methylotrophicus FR4B12 mostró ser la mejor antagonista de $B$. cinerea en hojas de vid, disminuyendo la densidad del fitopatógeno a partir de las $48 \mathrm{~h}$. Esta capacidad antagonista se ha encontrado en diversas cepas de la especie $B$. methylotrophicus, la cuales, en su mayoría, han sido aisladas de muestras de suelo y plantas. Algunas de estas cepas han demostrado tener actividad antagonista contra B. cinerea a nivel in vitro (Tumbarski, 2017), en planta (Ge et al., 2016) e inclusive junto con la aplicación de fungicidas químicos (Ji et al., 2019). Además, se ha observado que también tienen efecto contra otros fitopatógenos como son los hongos Magnaporthe oryzae (Shan et al., 2013) y Fusarium graminearum (Cheng et al., 2019); y la bacteria Ralstonia solanacearum (Almoneafy et al., 2012). Al igual que en otras especies del género Bacillus, se ha encontrado que cepas de $B$. methylotrophicus tienen la capacidad de producir lipopéptidos, como surfactinas, iturinas y fengicinas, las cuales son moléculas antimicrobianas de amplio espectro (Frikha-Gargouri et al., 2017), lo cual pudiera estar ocurriendo con la cepa B. methylotrophicus FR4B12 en estudio.

En conclusión, los resultados obtenidos en el presente trabajo demuestran la capacidad antagonista, la prevalencia e interacción microbiana de las cepas $B$. methylotrophicus FR4B12 y M. pulcherrima NB9 en hojas de vid. Lo anterior servirá como base para desarrollar estudios posteriores en campo que permitan determinar su potencial comercial como agentes de biocontrol.

\section{AGRADECIMIENTOS}

Los autores agradecen al Consejo Nacional de Ciencia y Tecnología CONACYTProblemas Nacionales, por el financiamiento del proyecto PDCPN 2016/3930.

\section{CONFLICTO DE INTERESES}

Los autores declaran que no existe conflicto de intereses. 


\section{REFERENCIAS}

Arriaga M. R., González H. M. A., Olalde P. V., Reyes R. B. G, Castillo G. A M., Pérez L. D. J. \& Aguilera G. L. I. 2012. Interrelación entre fósforo, Bacillus subtilis y Glomus fasciculatum con la calidad de Lilium. Фyton. 81: 59-68.

Aleti G., Lehner S., Bacher M., Compant S., Nikolic B., Plesko M., Schuhmacher R., Sessitsch A. \& Brader G. 2016. Surfactin variants mediate species-specific biofilm formation and root colonization in Bacillus. Environmental Microbiology. 18: 2634-2645.

Almoneafy A. A., Xie G. L., Tian W. X., Xu L. H., Zhang G. Q. \& Ibrahim M. 2012. Characterization and evaluation of Bacillus isolates for their potential plant growth and biocontrol activities against tomato bacterial wilt. African journal of Biotechnology. 11: 7193-7201.

AVQ, Asociación de Vitivinicultores de Querétaro. 2011. Estudio de impacto productivo de un viñedo. Ed. Asociación de Vitivinicultores de Querétaro, Querétaro. México. pp 111.

Bolivar A. H. J., Garrido C. \& Collado I.G. 2019. Endophytic microorganisms for biocontrol of the phytopathogenic fungus Botrytis cinerea. Phytochemistry Reviews. 2019: 1-20.

Amo F. J. 2018. El mercado del Vino en México. Estudios de mercado. ICEX España Exportaciones e $\quad$ inversiones. http://www.ivace.es/Internacional Informes-

Publicaciones/Pa\%C3\%ADses/M\%C3\%A9xico/Mexicovinoicex2018.pdf, (consultado Diciembre 7, 2019).

Cappelletti M. Perazzolli M., Antonielli L., Nesler A., Torboli E., Biachedi P. L., Massimo P., Puopolo G. \& Pertot L. 2016. Leaf treatments with a protein-based resistance inducer partially modify phyllosphere microbial communities of grapevine. Frontiers in Plant Science. 7(1053): 1-7.

Cheng X., Ji X., Ge Y., Li J., Qi W. \& Qiao K. 2019. Characterization of antagonistic Bacillus methylotrophicus isolated from rhizosphere and its biocontrol effects on maize stalk rot. Phytopathology. 109: 571-581.

Cray J. A., Connor M. C., Stevenson A., Houghton J. D., Rangel D. E., Cooke L. R. \& Hallsworth, J. E. 2016. Biocontrol agents promote growth of potato pathogens, depending on environmental conditions. Microbial biotechnology. 9(3): 330-354.

Dukare A. S., Paul S., Nambi V. E., Gupta R. K., Singh R., Sharma K. \& Vishwakarma R. K. 2019. Exploitation of microbial antagonists for the control of postharvest diseases of fruits: a review. Critical Reviews In Food Science and Nutrition. 59: 1498-1513. 
Ferraz P., Cássio F. \& Lucas C. 2019. Potential of yeasts as biocontrol agents of the phytopathogen causing cacao Witches' Broom Disease: is microbial warfare a solution? Frontiers in Microbiology. 10: 1-13.

Frikha-Gargouri O., Ben Abdallah D., Ghorbel I., Charfeddine I., Jlaiel L., Triki M. A. \& Tounsi, S. 2017. Lipopeptides from a novel Bacillus methylotrophicus 39b strain suppress Agrobacterium crown gall tumours on tomato plants. Pest Management Science. 73: 568-574.

Ge B., Liu B., Nwet T. T. Zhao, W. Shi L. \& Zhang, K. 2016. Bacillus methylotrophicus strain NKG-1, isolated from Changbai Mountain, China, has potential applications as a biofertilizer or biocontrol agent. PloS one. 11: 1-13.

Gianotti A., Sacchetti G., Guerzoni M. E. \& Dalla Rosa, M. 2001. Microbial aspects on short-time osmotic treatment of kiwifruit. Journal of Food Engineering. 49: 265270.

Guerrero P. V. M., Jacobo C. J. L., Parra Q. R. A., Linares M. M. I., Ojeda B. D. L., Hernández R. O. A., Robles H. L., Berlanga R. D. \& Cabanillas M. I. J. 2019. Botrytis cinerea Pers. en manzana poscosecha, control con Candida oleophila Montrocher y/o fungicidas sintéticos. Nova Scientia. 11(22):69-84.

Ji X., Li J., Meng Z., Zhang S., Dong B. \& Qiao, K. 2019. Synergistic effect of combined application of a new fungicide fluopimomide with a biocontrol agent Bacillus methylotrophicus TA-1 for management of gray mold in tomato. Plant disease. 103(8): 1991-1997.

Keller M. 2015. The Science of Grapevines: Anatomy and Physiology. Academic Press. Prosser, WA. USA. pp 522.

Khazaeli P., Zamanizadeh H., Morid B. \& Bayat, H. 2012. Morphological and molecular identification of Botrytis cinerea causal agent of gray mold in rose greenhouses in centeral regions of Iran. International Journal of Agricultural Science and Research. 1:19-24.

López-González R. C., Mendoza M.G., Martínez R. A. \& Pacheco, J.R. 2019. Actividad antagonista de Metschnikowia pulcherrima NB9 y Bacillus methylotrophicus FR4B12 contra Botrytis cinerea. In: VI Encuentro Internacional Sobre Biotecnología En La UAT. Tlaxcala, México.

Lúquez B. C. V., Formento J. C. \& Díaz P. E. 2007. Las yemas, principal reservorio de levaduras entre los órganos aéreos vegetativos y reproductivos de la vid (Vitis vinifera var. Malbec). Revista de la Facultad de Ciencias Agrarias. 39(1):83-91.

Martínez L. L., Martínez P. R.A., Hernández I. M., Arvizu M. S. M. \& Pacheco A. J. R. 2013. Caracterización de rizobacterias aisladas de tomate y su efecto en el crecimiento de tomate y pimiento. Revista Fitotecnia Mexicana. 36(1): 63-69.

Pauluk-Correa I., De Almeida A. B. \& Pimentel I. C. 2018. Biocontrol activity of yeast strains isolated from green coffee beans against ochratoxin a-producing 
Aspergillus species. International Journal of Microbiological Research. 10(6): 12681273.

Pande S., Galloway J., Gaur P. M., Siddique K. H. M., Tripathi H. S., Taylor P., MacLeod M. W. J., Basandrai A. K., Bakr A. \& Joshi, S. 2006. Botrytis grey mould of chickpea: a review of biology, epidemiology, and disease management. Australian Journal and Agricultural Research. 57(11):1137-1150.

Pandin C., Le Coq D., Canette A., Aymerich S. \& Briandet, R. 2017. Should the biofilm mode of life be taken into consideration for microbial biocontrol agents? Microbial Biotechnology. 10: 719-734.

Perazzolli M., Antonielli L., Storari M., Puopolo G., Pancher M., Giovannini O., Pindo M. \& Pertot L. 2014. Resilience of the natural phyllosphere microbiota of the grapevine to chemical and biological pesticides. Applied and Environmental Microbiology. 80(12):3585-3596.

Petrasch S., Silva C. J., Mequida-Pesci S. D., Gallegos K., Van den Abeele C., Papin V., Fernandez-Acero F. J., Knapp S. J. \& Blanco-Ulate B. 2019. Infection strategies deployed by Botrytis cinerea, Fusarium acuminatum, and Rhizopus stolonifer as a function of tomato fruit ripening stage. Frontiers in Plant Science. 10:223:1-17.

Rosslenbroich H. J. \& Stuebler, D. 2000. Botrytis cinerea-history of chemical control and novel fungicides for its management. Crop Protection. (8-10)19: 557561.

Rupp S., Weber R. W. S., Rieger D., Detzel P. \& Hahn M. 2017. Spread of Botrytis cinerea strains with multiple fungicide resistence in German Horticulture. Frontiers in Microbiology.1(7):1-12.

Shan H., Zhao M., Chen D., Cheng J., Li J., Feng Z., Ma Z. \& An D. 2013. Biocontrol of rice blast by the phenaminomethylacetic acid producer of Bacillus methylotrophicus strain BC79. Crop Protection. 44: 29-37.

Silva F. D. J., Ferreira L. C., Campos V. P., Cruz-Magalhães V., Barros A. F., Andrade, J. P. \& De Souza J. T. 2019. Complete genome sequence of the biocontrol agent Bacillus velezensis UFLA258 and its comparison with related species: diversity within the commons. Genome Biology and Evolution. 11: 28182823.

Tumbarski Y. 2017. Antimicrobial activity of Bacillus methylotrophicus BM47 against Botrytis cinerea in different conditions of cultivation. Bulgarian Journal of Veterinary Medicine. 20:89-94.

Vionnet L., De Vrieze M., Dutartre A., Gfeller A., Luthi A.,L'Haridon F. \& Weisskopf L. 2018. Microbial life in the grapevine: what can we expect from the leaf microbiome? OENO One 52(3): 219-224. 
Wei F., Hu X. \& Xu X. 2016. Dispersal of Bacillus subtilis subtilis and its effect on strawberry phyllosphere microbiota under open field and protection conditions. Scientific reports. 6(1):1-9.

Whiteman S. A. \& Stewart A. 1998. Supression of Botrytis cinerea sporulation on irradiated grape leaf tissue by the antagonistic bacterium Serratia liquefaciens. New Zeland Journal of Crop and Horticultural Science. 26:325-330.

Wu B., Wang X., Yang L., Yang H., Zeng H., Qu Y., Wang C., Yu J., Li J., Xu D., He Z. \& Chen S. 2016. Effects of Bacillus amyloliquefaciens ZM9 on bacterial wilt and rhizosphere microbial communities of tobacco. Applied Soil Ecology. 103:1-12.

Zhang B., Bai Z., Hoefel D., Tang L., Yang Z., Zhuang G., Jianzhou Y. \& Zhang H. 2008. Assessing the impact of the biological control agent Bacillus thuringiensis on the indigenous microbial community within the pepper plant phyllosphere. FEMS Microbiology Letters. 284:102-108.

Zhang H., Mahunu G. K., Castoria R., Apaliya M.T. \& Yang Q. 2017. Augmentation of biocontrol agents with physical methods against postharvest diseases of fruits and vegetables, Trends in Food Science \& Technology. 69: 36-45. 